\title{
Interpretable Learning Approaches in Resting-State Functional Connectivity Analysis: The Case of Autism Spectrum Disorder
}

\author{
Jinlong Hu $\mathbb{D}^{1,2}$ Lijie Cao $\mathbb{D}^{1,2}$ Tenghui Li $\mathbb{D}^{1,2}$ Bin Liao $\mathbb{D}^{3},{ }^{3}$ Shoubin Dong $\mathbb{D},{ }^{1,2}$ and Ping $\mathrm{Li}^{4}$ \\ ${ }^{1}$ School of Computer Science and Engineering, South China University of Technology, Guangzhou, China \\ ${ }^{2}$ Communication and Computer Network Laboratory of Guangdong, South China University of Technology, Guangzhou, China \\ ${ }^{3}$ College of Mathematics and Informatics, South China Agricultural University, Guangzhou, China \\ ${ }^{4}$ Faculty of Humanities, The Hong Kong Polytechnic University, Hong Kong, China
}

Correspondence should be addressed to Jinlong Hu; jlhu@scut.edu.cn

Received 26 March 2020; Accepted 5 May 2020; Published 18 May 2020

Guest Editor: Lin Lu

Copyright (c) 2020 Jinlong $\mathrm{Hu}$ et al. This is an open access article distributed under the Creative Commons Attribution License, which permits unrestricted use, distribution, and reproduction in any medium, provided the original work is properly cited.

\begin{abstract}
Deep neural networks have recently been applied to the study of brain disorders such as autism spectrum disorder (ASD) with great success. However, the internal logics of these networks are difficult to interpret, especially with regard to how specific network architecture decisions are made. In this paper, we study an interpretable neural network model as a method to identify ASD participants from functional magnetic resonance imaging (fMRI) data and interpret results of the model in a precise and consistent manner. First, we propose an interpretable fully connected neural network (FCNN) to classify two groups, ASD versus healthy controls (HC), based on input data from resting-state functional connectivity (rsFC) between regions of interests (ROIs). The proposed FCNN model is a piecewise linear neural network (PLNN) which uses piecewise linear function LeakyReLU as its activation function. We experimentally compared the FCNN model against widely used classification models including support vector machine (SVM), random forest, and two new classes of deep neural network models in a large dataset containing 871 subjects from ABIDE I database. The results show the proposed FCNN model achieves the highest classification accuracy. Second, we further propose an interpreting method which could explain the trained model precisely with a precise linear formula for each input sample and decision features which contributed most to the classification of ASD versus HC participants in the model. We also discuss the implications of our proposed approach for fMRI data classification and interpretation.
\end{abstract}

\section{Introduction}

Autism spectrum disorder (ASD) is a subtype of extensive developmental disorder which is characterized by reciprocal social communication impairment as well as repetitive, restricted, and stereotyped behaviors [1]. The cause of ASD is uncertain, and the diagnosis is often difficult since the expressions of ASD symptoms are diverse and may vary over the course of development [2]. Functional magnetic resonance imaging (fMRI) is one of the most widespread approaches which is noninvasive and useful for understanding brain function [3]. fMRI has been applied recently for distinguishing ASD patients from healthy controls, and vari- ous machine learning methods have been used to analyze fMRI data of brain disorder [4-7]. However, so far, it has been challenging to analyze fMRI data for brain disorder due to the data characteristics such as high dimensionality, structural complexity, nonlinear separability, and the sequential changes of traceable signals in each voxel [8].

Given the excellent learning capability and classification performance in many domains, deep learning methods have been recently applied to fMRI data from ASD patients [9-14]. Sólon et al. [9] investigated the patterns of functional connectivity that help to identify ASD participants from functional brain imaging data. They used stacked denoising autoencoders for the unsupervised pretraining 
stage to extract a low-dimensional version from the ABIDE database and then applied the encoder weights to a multilayer perceptron for classification. The ABIDE (Autism Brain Imaging Data Exchange) database [15] contains a rich set of fMRI data that has aggregated functional and structural brain imaging data collected from multisite around the world (see Section 2.1 below for details). Guo et al. [10] stacked multiple sparse autoencoders for data dimension reduction and developed a feature selection method to select features with high discriminative power. Then, they used a softmax regression on top of the stacked sparse autoencoders for data classification. Eslami et al. [11] used an autoencoder and a single-layer perceptron to extract lower dimensional features, and the trained perceptron is used for the final round of classification. Brown et al. [12] proposed an element-wise layer based on BrainNetCNN [16] and used anatomically informed, data dependent, prior to regularize the weights of the layer.

Researchers are also trying to explain these models, by analyzing the discriminative features or potential neuroimaging biomarkers that contribute to the classification of ASD from healthy controls. Li et al. [17] trained a deep neural network to classify 3D fMRI volumes, developed a frequencynormalized sampling method to replace a ROI of the original image with the sampling data, and put it in the trained model to get a new prediction. Based on the different predicting performance, they used a statistical method to interpret the importance of the ROI. In the study of discovering imaging biomarkers for ASD [18], they went beyond looking at only individual features by using Shapley value explanation on interactive features' prediction power analysis. Guo et al. [10] proposed a deep neural network with a feature selection method from multiple trained sparse autoencoders, then developed Fisher's score-based biomarker identification method for their deep neural network using the rs-fMRI dataset in ABIDE I. These approaches all led to useful insights into the mechanism of deep learning models. However, such deep and nonlinear models are usually constructed as black boxes with complex network structure and hidden internal logic and are difficult to interpret with regard to how architecture decisions are consistently made by researchers [19].

In this study, we introduce an interpretable learning approach for resting-state functional connectivity analysis. We firstly propose an interpretable neural network model to distinguish between ASD participants and healthy controls (HC) based on resting-state functional connectivity (rsFC) of each subject. The proposed model is an interpretable fully connected neural network (FCNN), which uses piecewise linear function LeakyReLU as its activation function. It is a fully connected neural network including two hidden layers, input layer and output layer. Further, the proposed model is a piecewise linear neural network (PLNN) [20], which is mathematically equivalent to a set of local linear classifiers and could be interpreted precisely and consistently [19]. Secondly, taking advantage of the interpretation of PLNN, we propose an interpretable method which could explain the trained classification model with a precise linear formula for each input sample and the decision features which contribute most to classify ASD versus $\mathrm{HC}$ in the model.

We experimentally compared the proposed FCNN model against widely used benchmark models including SVM, random forest (RF), and two new neural network models in classifying data from the multisite ABIDE I database [15]. The proposed FCNN model, based on input data from rsFC between regions of interests (ROIs) accord to the AAL atlas [21], achieved the highest accuracy $69.81 \%$ in the large dataset containing 871 subjects (403 ASD patients and 468 healthy controls). We also explained the most important features in the model.

\section{Dataset and Preprocessing}

2.1. Dataset. We chose the dataset from the Autism Brain Imaging Data Exchange (ABIDE) initiative [15] to confirm the approach proposed in this study. The ABIDE initiative has aggregated functional and structural brain imaging data collected from multiple sites around the world. The dataset used in this study contained 871 subjects acquired from 17 acquisition sites with different imaging protocols that met the imaging quality and phenotypic information criteria [22]. This dataset includes 403 individuals suffering from ASD and 468 healthy controls (HC).

2.2. Preprocessing. We downloaded the preprocessed restingstate fMRI data from the Preprocessed Connectomes Project (PCP) (http://preprocessed-connectomes-project.org/abide/ download.html). The data [23] was preprocessed by the Configurable Pipeline for the Analysis of Connectomes (CPAC) pipeline that included the following procedure: slice timing correction, motion realignment, intensity normalization, regression of nuisance signals, band-pass filtering (0.01-0.1 Hz), and registration of fMRI images to standard anatomical space (MNI152). The detailed description of pipeline can be found at http://preprocessed-connectomesproject.org/abide/Pipelines.html. The data was parcellated into 116 regions of interests (ROIs) using the AAL atlas [21].

\section{Proposed Approach}

The flow chart of the proposed interpretable learning approach is shown in Figure 1. First, we propose the FCNN model for classifying ASD and healthy participants, including extracting the rsFC features, training the FCNN model, and validating the model. Second, we interpret the trained model with an easily explained linear formula for each subject, identifying the decision rsFC features for the ASD group from the data.

3.1. Feature Extraction. The resting-state fMRI data was preprocessed as described in Section 2. The brain was parcellated into 116 regions of interests (ROIs) according to the AAL atlas [21]. Then, the mean time series of each ROI was extracted for each subject, and the rsFCs between ROIs were measured by computing Pearson's correlation coefficient of the extracted time series. A $116 \times 116$ connectivity matrix was constructed for each subject, respectively. 


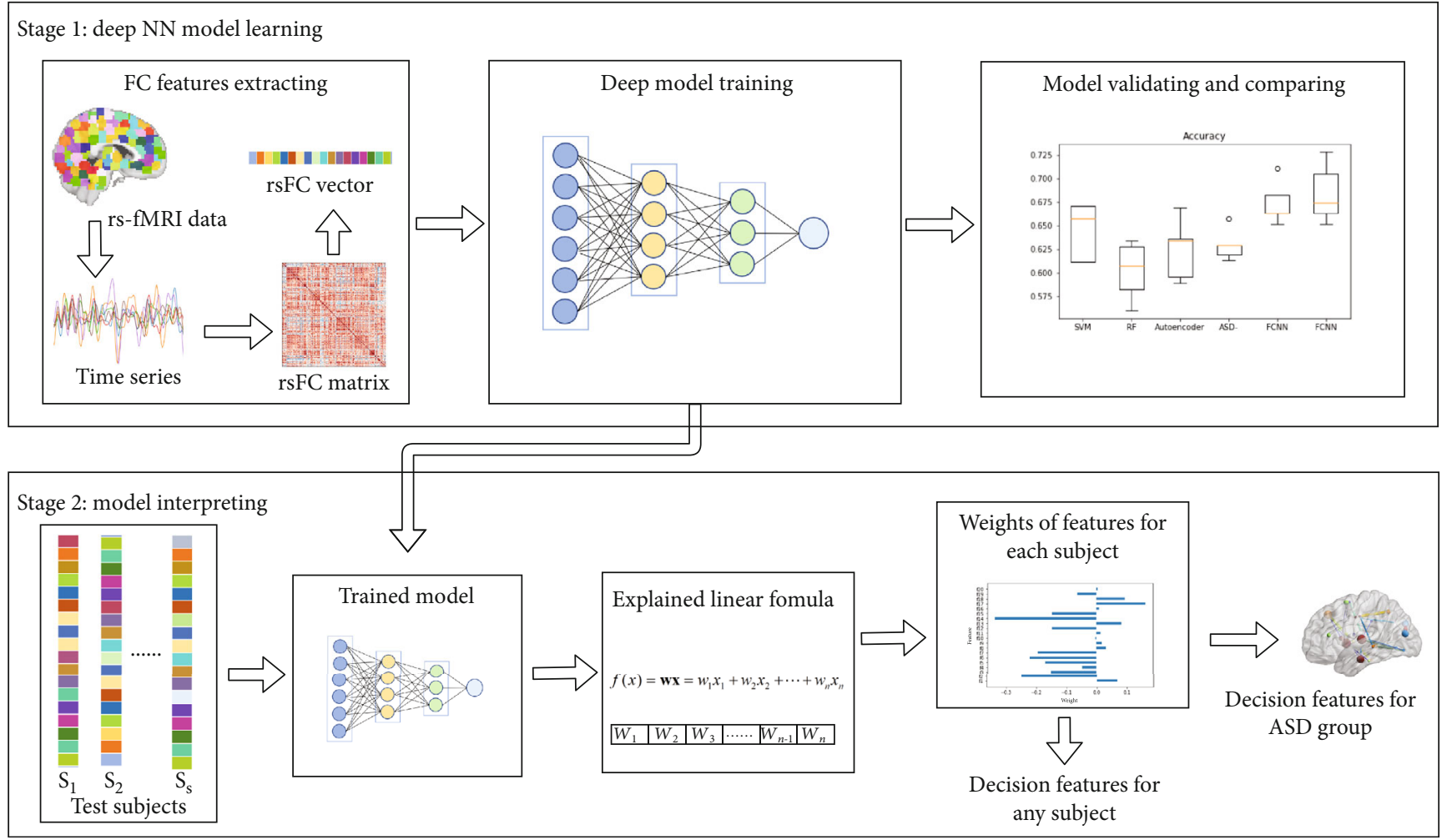

FIGURE 1: Flow chart of the proposed approach: learning and interpreting model on resting-state fMRI data.

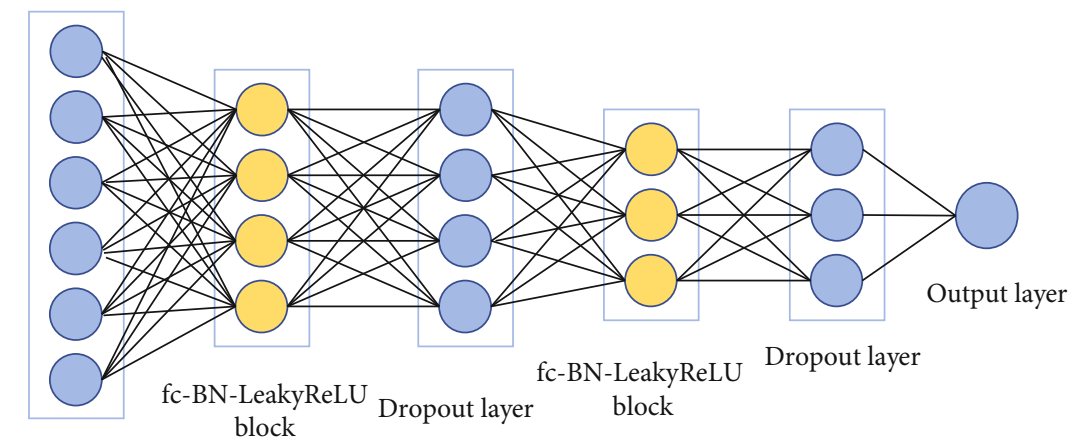

Input layer

FIgURE 2: The architecture of the proposed FCNN model.

Fisher transformation was applied to the connectivity matrices to improve normality. The upper triangle values were then extracted and flattened into vectors, with the dimension of the feature vector which is $(116 \times(116-1)) /$ $2=6670$.

3.2. FCNN Model. The architecture of the proposed FCNN model is shown in Figure 2. The FCNN is a fully connected neural network and a piecewise linear neural network (PLNN), where the PLNN is a deep neural network in which the nonlinear activation function is a piecewise linear function with a constant number of pieces [20].

The FCNN model contains two fc-BN-LeakyReLU blocks, where the fc-BN-LeakyReLU block consists of a fully connected (fc) layer followed by a Batch Normalization (BN) layer and LeakyReLU activation function.

LeakyReLU is a variant of rectified linear unit (ReLU) [24] which allows a small, positive gradient when the unit is not active [25]. For each hidden neuron $u$, LeakyReLU is defined as

$$
f(u)= \begin{cases}u, & u \geq 0, \\ \alpha u, & u<0,\end{cases}
$$

where $\alpha$ represents slope coefficient. LeakyReLU is clearly a piecewise linear function.

In this study, for simplicity and clarity, we regarded a fcBN-LeakyReLU block as a hidden layer. For a model with $L$ 
layers, a fc layer can be formulated as

$$
z^{(l+1)}=W^{(l)} a^{(l)}+b^{(l)},
$$

where $l \in\{1, \cdots, L-1\}$; suppose there are $n$ neurons in layer $l$ and $m$ neurons in layer $l+1, W^{(l)}$ is $m \times n$ weight matrix, $b^{(l)}$ is $m \times 1$ bias vector, and $a^{(l)}$ will be in Equation (3).

Then, the fc-BN-LeakyReLU block can be written as

$$
a^{(l)}=f\left(\mathrm{BN}\left(z^{(l)}\right)\right)
$$

where $l \in\{2, \cdots, L-1\}$ are hidden layers, $f(\bullet)$ is the LeakyReLU function, explicitly, and $a^{(1)}$ is the input instance $x$.

The sigmoid function is applied on the output layer to predict the probability of any given participant being an ASD patient. The number of units (nodes) is 6670, 64, 32 , and 1, respectively, for input layer, two fully connected layers, and output layer. The dropout layer is added to avoid data overfitting, and the loss function uses binary cross entropy.

3.3. Interpreting Method. We interpret the trained neural network model with two stages: (i) computing the decision boundary of a fixed instance and the weight of features in linear formula for the instance and (ii) extracting and analyzing decision features of the trained model in the ASD group level.

In the first stage, we computed the decision boundary of a fixed instance $x$.

For each hidden neuron $u, \mathrm{BN}$ can be formulated as

$$
y=\frac{\gamma}{\sqrt{\operatorname{Var}[u]+\epsilon}} \cdot u+\left(\beta-\frac{\gamma \mathrm{E}[u]}{\sqrt{\operatorname{Var}[u]+\epsilon}}\right)
$$

where $\gamma$ and $\beta$ are learned parameters [26]. In the test phase of the model, $\operatorname{Var}[u]$ and $E[u]$ are fixed, so Equation (4) can be regarded as a linear function.

As shown in Equation (1), for hidden neurons with LeakyReLU activation function, there are two kinds of activation status that each corresponds to a corresponding linear function where the mapping relationship between $f(u)$ and $u$ is linear. And it is proved that for a fixed PLNN model and a fixed instance $x$, the output of model $F(x)$ on an input $x$ can be seen as a linear classifier [19], which can be formulated as

$$
F(x)=\operatorname{sigmoid}(\widehat{W} x+\widehat{b})
$$

where $\widehat{W}=\prod_{h=0}^{L-3} \tilde{W}^{(L-1-h)} W^{(1)}$ is the coefficient vector of $x$ and $\widehat{b}$ is the constant intercept. For a fixed input instance $x$, $F(x)$ is a linear classifier whose decision boundary is explicitly defined by $\widehat{W} x+\widehat{b}$. Therefore, $\widehat{W}$ are weights assigned to the features of $x$.

As for FCNN, we computed $\tilde{W}$ as follows: since $\mathrm{BN}$ can be regarded as a linear function in the test phase of model as discussed above, the Equation (3) can be rewrit- ten as

$$
a^{(l)}=f\left(\tilde{\gamma}^{(l)} \circ z^{(l)}+\tilde{\beta}^{(l)}\right)
$$

where $\tilde{\gamma}^{(l)}$ is the constant slope, $\tilde{\beta}^{(l)}$ is the constant intercept, for all $l \in\{2, \cdots, L-1\}$. Since $f(\bullet)$ is the piecewise linear activation function, Equation (6) can be rewritten as

$$
a^{(l)}=r^{(l)} \circ\left(\tilde{\gamma}^{(l)} \circ z^{(l)}+\tilde{\beta}^{(l)}\right)+t^{(l)},
$$

where $r^{(l)}$ is the constant slope and $t^{(l)}$ is the constant intercept. By plugging Equation (7) into Equation (2), we rewrite $z^{(l+1)}$ as

$$
\begin{aligned}
z^{(l+1)} & =W^{(l)}\left(r^{(l)} \circ\left(\tilde{\gamma}^{(l)} \circ z^{(l)}+\tilde{\beta}^{(l)}\right)+t^{(l)}\right)+b^{(l)} \\
& =\tilde{W}^{(l)} z^{(l)}+\tilde{b}^{(l)}
\end{aligned}
$$

where $\tilde{W}^{(l)}=W^{(l)} \circ r^{(l)} \circ \tilde{\gamma}^{(l)}$ is an extended version of the Hadamard product.

In the second stage, based on the weights $\widehat{W}$ for features of each test instance $x$, we could get the top $K$ features with the highest weight. Then, we count the number of occurrences $n^{f}$ of feature $f$ in the top-k-feature-set from all the instances. By setting a threshold on $n^{f}$, we can get decision feature set $F$ which contributes most to classify ASD versus $\mathrm{HC}$ in the model.

The whole flow of the interpreting method is formulated as in Algorithm 1. We firstly obtain the top-k-feature-set $F_{K}^{X}$ for each instance $x$, and then, we obtain the decision feature set $F$ by selecting the feature $f$ whose occurrence number as a percentage of total instances is greater to the parameter $\varepsilon$. Meanwhile, we could also get the weights of all features for any specified test instance, which could help to explain the decision made by the trained model for the instance.

\section{Classification Experiments}

With the above approach and the model architecture, we conducted experiments on the ABIDE I dataset with 871 subjects and applied the interpretation algorithm to explain the results.

To evaluate the performance of the proposed method, we use sensitivity, specificity, accuracy, F1, and AUC as our metrics. These metrics are defined as follows:

$$
\begin{aligned}
\text { sensitivity } & =\frac{\mathrm{TP}}{\mathrm{TP}+\mathrm{FN}}, \\
\text { specificity } & =\frac{\mathrm{TN}}{\mathrm{TN}+\mathrm{FP}}, \\
\text { accuracy } & =\frac{\mathrm{TP}+\mathrm{TN}}{\mathrm{TP}+\mathrm{FN}+\mathrm{TN}+\mathrm{FP}}, \\
\mathrm{F} 1 & =\frac{2 \bullet \mathrm{TP}}{2 \cdot \mathrm{TP}+\mathrm{FP}+\mathrm{FN}},
\end{aligned}
$$


Input: a well-trained FCNN; the set of test instances $D$; parameter $K$, the number of top important features of instance $x$; parameter $\varepsilon$, number of occurrences of feature $f$ as a percentage of total instances.

Output: decision feature set $F$

1. Initialization: $F=\varnothing, F_{K}^{D}=\varnothing$

2. For each $x \in D$ do

3. Compute the weight $\widehat{W}$

4. Get the $K$ top features $F_{K}^{X}$ with the highest weight

5. $F_{K}^{D} \leftarrow F_{K}^{D} \cup F_{K}^{X}$

6. End for

7. For each feature $f$ in $F_{K}^{D}$

8. Count the number of occurrences $n^{f}$ of feature $f$

9. If $n^{f}>|D| * \varepsilon$

10. $F \leftarrow F \cup f$

11. End for

12. Return $F$

Algorithm 1. A run-down flow for trained model interpreting.

TABLE 1: Classification performance using 5-fold cross-validation (mean \pm std).

\begin{tabular}{lccccc}
\hline & Accuracy & Sensitivity & Specificity & F1 & AUC \\
\hline SVM-linear & $0.6441 \pm 0.0281$ & $0.5856 \pm 0.0238$ & $0.6946 \pm 0.0556$ & $0.6039 \pm 0.0219$ & $0.7053 \pm 0.0372$ \\
SVM-rbf & $0.6624 \pm 0.0283$ & $0.5631 \pm 0.0623$ & $0.7478 \pm 0.0629$ & $0.6055 \pm 0.0403$ & $0.7059 \pm 0.0283$ \\
RF & $0.6326 \pm 0.0416$ & $0.4590 \pm 0.0428$ & $0.7821 \pm 0.0442$ & $0.5364 \pm 0.0506$ & $0.6790 \pm 0.0339$ \\
Autoencoder+MLP [9] & $0.6717 \pm 0.0217$ & $0.6225 \pm 0.1601$ & $0.7140 \pm 0.1124$ & $0.6259 \pm 0.0784$ & $0.6682 \pm 0.0293$ \\
ASD-DiagNet [11] & $0.6900 \pm 0.0172$ & $0.6277 \pm 0.0642$ & $0.7436 \pm 0.0299$ & $0.6504 \pm 0.0338$ & $0.6857 \pm 0.0201$ \\
FCNN (without BN) & $0.6889 \pm 0.0109$ & $0.6204 \pm 0.0844$ & $0.7479 \pm 0.0624$ & $0.6456 \pm 0.0378$ & $0.7099 \pm 0.0227$ \\
FCNN & $0.6981 \pm 0.0169$ & $0.6305 \pm 0.0474$ & $0.7563 \pm 0.0182$ & $0.6582 \pm 0.0287$ & $0.7262 \pm 0.0308$ \\
\hline
\end{tabular}

where TP is defined as the number of ASD subjects that are correctly classified, FP is the number of normal subjects that are misclassified as ASD subjects, TN is defined as the number of normal subjects that are correctly classified, and FN is defined as the number of ASD subjects that are misclassified as normal subjects. Specifically, sensitivity measures the proportion of ASD subjects that are correctly identified as such; specificity measures the proportion of normal subjects that are correctly identified as such. AUC is defined as the area under the Receiver Operating Characteristic (ROC) curve.

4.1. Comparison Models. Given the above FCNN model, we use the following models as benchmarks for comparison.

SVM: support-vector machine (SVM) model with linear kernel and rbf kernel. The SVM method has been widely used to classify fMRI data for brain disorders. The parameters are chosen by grid search.

RF: random forest (RF) is an ensemble learning method for classification. The parameters are chosen by grid search.

Autoencoder+MLP: the model was proposed by Sólon et al. [9]. Two stacked denoising autoencoders are pretrained; then, the encoder weights are applied to a multilayer perceptron (MLP), and the MLP is fine tuned to predict the proba- bility of any given participants being ASD. We applied the encoder weights to the MLP with the configuration: 66701000-600-2.

ASD-DiagNet: this method is proposed by Eslami et al. [11]. An autoencoder is used to extract a lower dimensional feature representation. Then, the feature representation is fed into a single-layer perceptron (SLP) with sigmoid function for classification. The autoencoder and SLP classifier are trained simultaneously. The input layer and output layer have 6670 units fully connected to a bottleneck of 1667 units from the hidden layer. Data augmentation using EROS similarity measure is applied with 5 nearest neighbors of each sample.

FCNN: the proposed FCNN model as described above in Figure 2. The model contains two fully connected layers: the first layer has 64 units and the second layer has 32 units. The dropout ratio is set to 0.8 . We used the Adam optimizer with a learning rate of 0.0005 .

For autoencoder+MLP [9] and ASD-DiagNet [11], we used their online code to evaluate the models.

All functional connectivity features are flattened into one dimensional vector (see Figure 1), and the vectors are inputs in all model for training and classification. All the models were trained with 6670 functional connectivity features for each subject. We employed a 5-fold cross-validation setting 


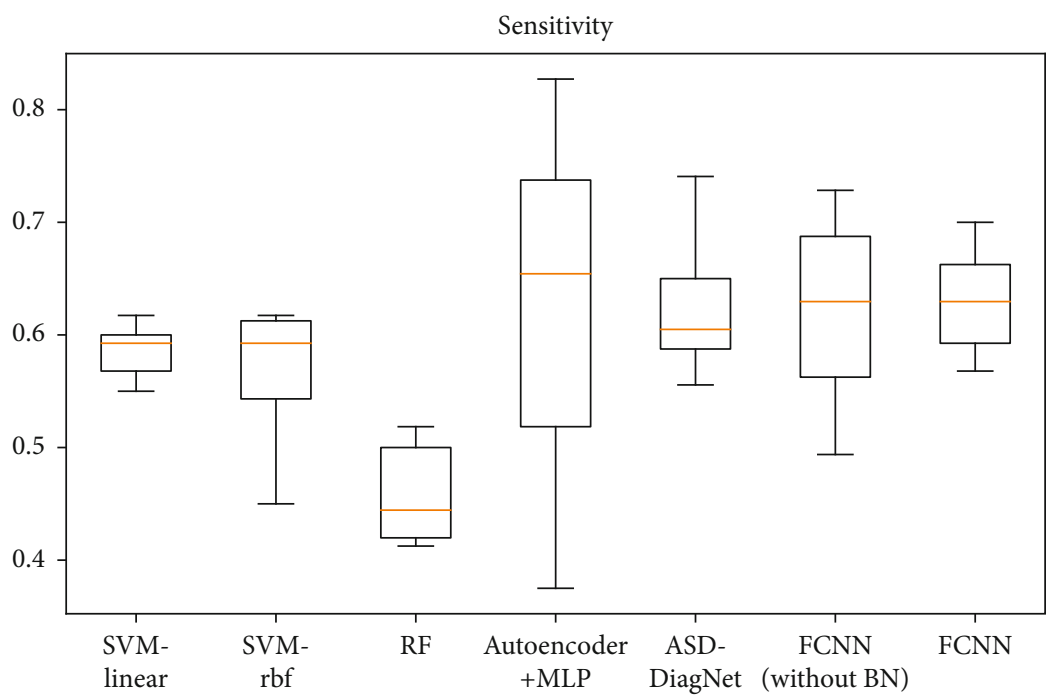

(a)

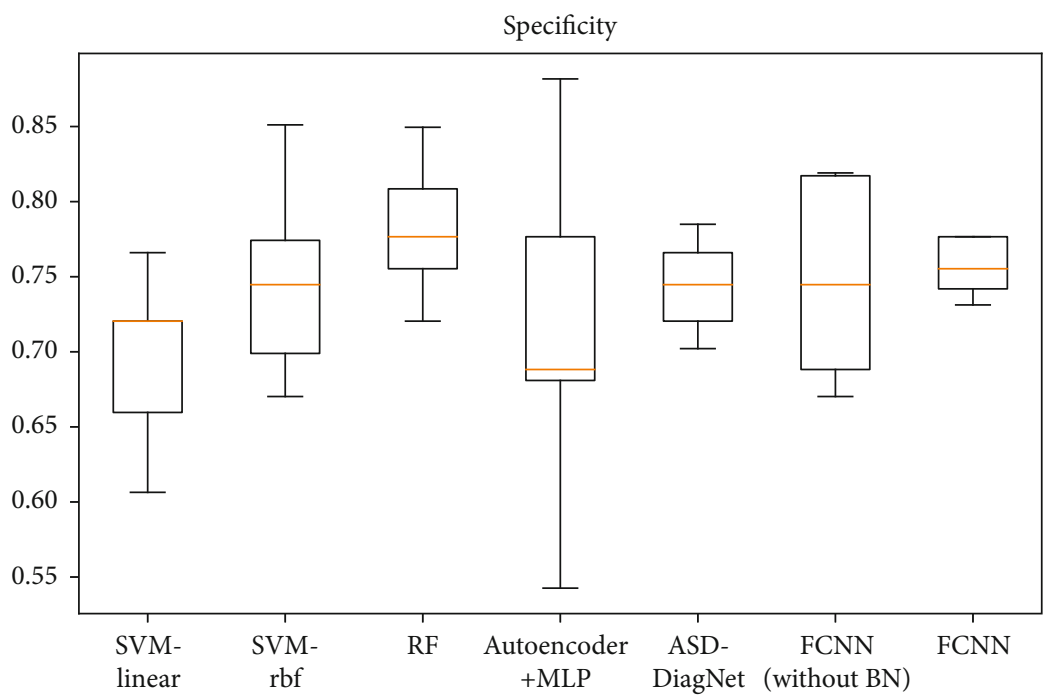

(b)

F1

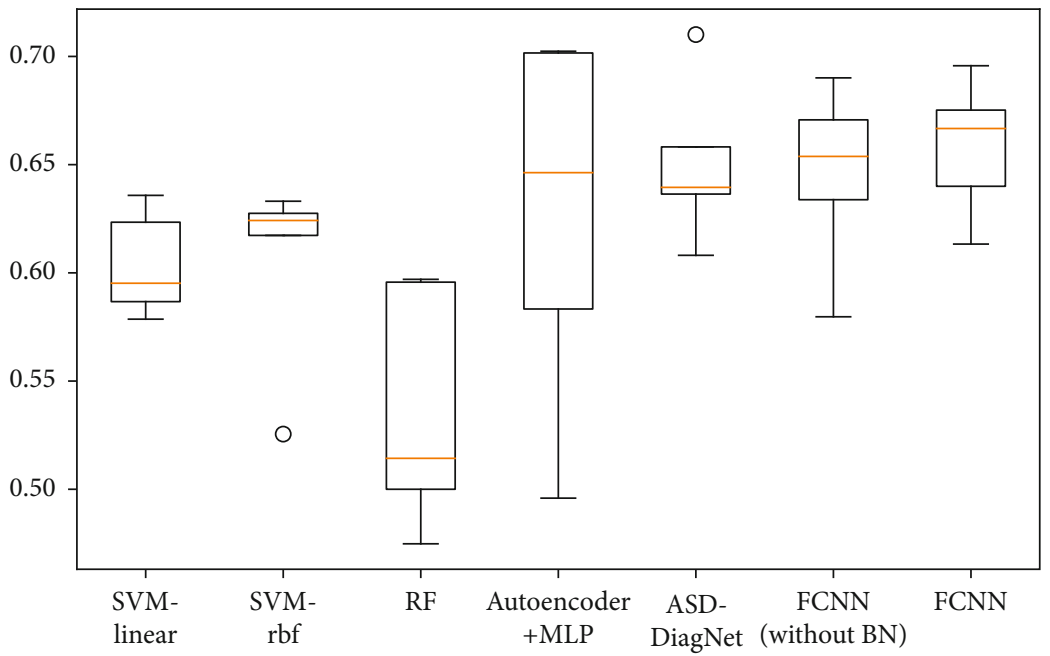

(c)

Figure 3: Continued. 


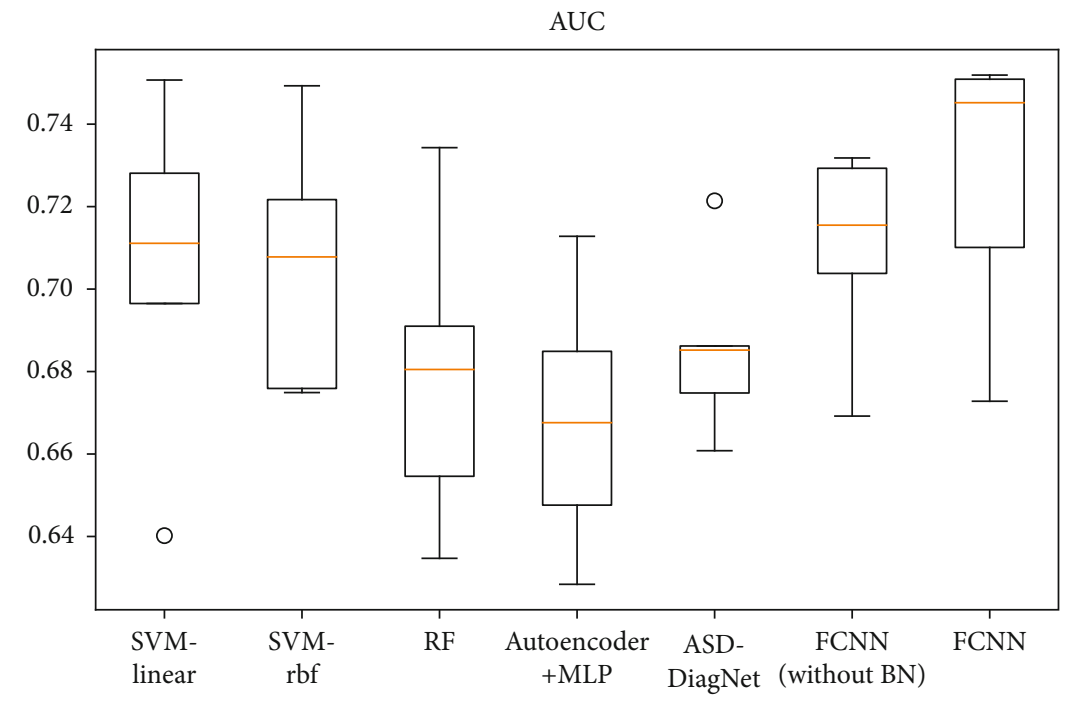

(d)

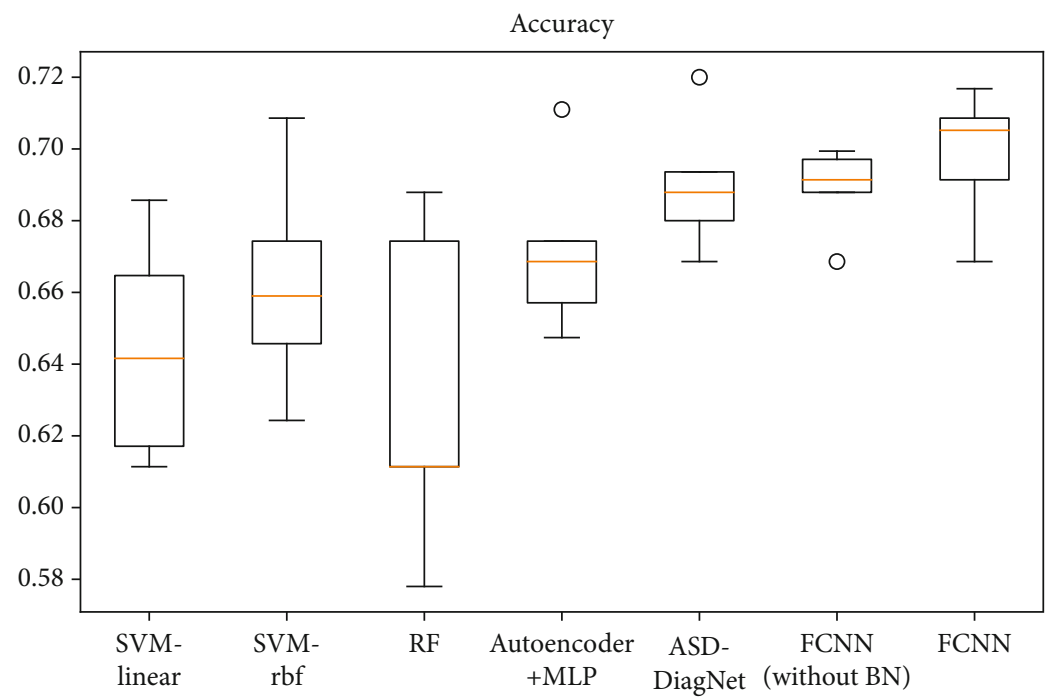

(e)

Figure 3: (a) Sensitivity, (b) specificity, (c) F1, (d) AUC, and (e) accuracy for classification task.

to evaluate the performance of all the models. The experiments were carried out on all 871 subjects including both ASD patients and healthy controls.

4.2. Classification Results. The classification results are shown in Table 1 and Figure 3. Box plots for sensitivity, specificity, F1, AUC, accuracy for classification task using 5-fold crossvalidation are shown in Figure 3, where the middle line in each box represents the median value, and the circle represents the outlier.

The proposed FCNN model achieved the best performance on most evaluation metrics with accuracy of $69.81 \%$, sensitivity of $63.05 \%$, specificity of $75.63 \%$, F1 of $65.82 \%$, and AUC of 0.7262 . The results showed that the deep learning models (FCNN, autoencoder+MLP, and ASD-DiagNet) have the better classification performance in general than the traditional methods (SVM and RF) on the resting-state fMRI dataset. As for the method autoencoder+MLP [9], we would like to mention that they reported $70 \%$ accuracy in their paper; the performance we reported is not as good as theirs, maybe because the brain atlas we used is different.

We also compared the FCNN model with or without the BN (Batch Normalization) layer in Table 1. The results showed that the BN layer improves the performance and stability of the model.

\section{Interpretation Experiments and Analysis}

5.1. Model Interpreting for an Instance. According to Section 3.3, for a trained FCNN model and any instance $x$ with $n$ features, $x=\left\{x_{1}, x_{2}, \cdots, x_{n-1}, x_{n}\right\}$, the fixed model can be 


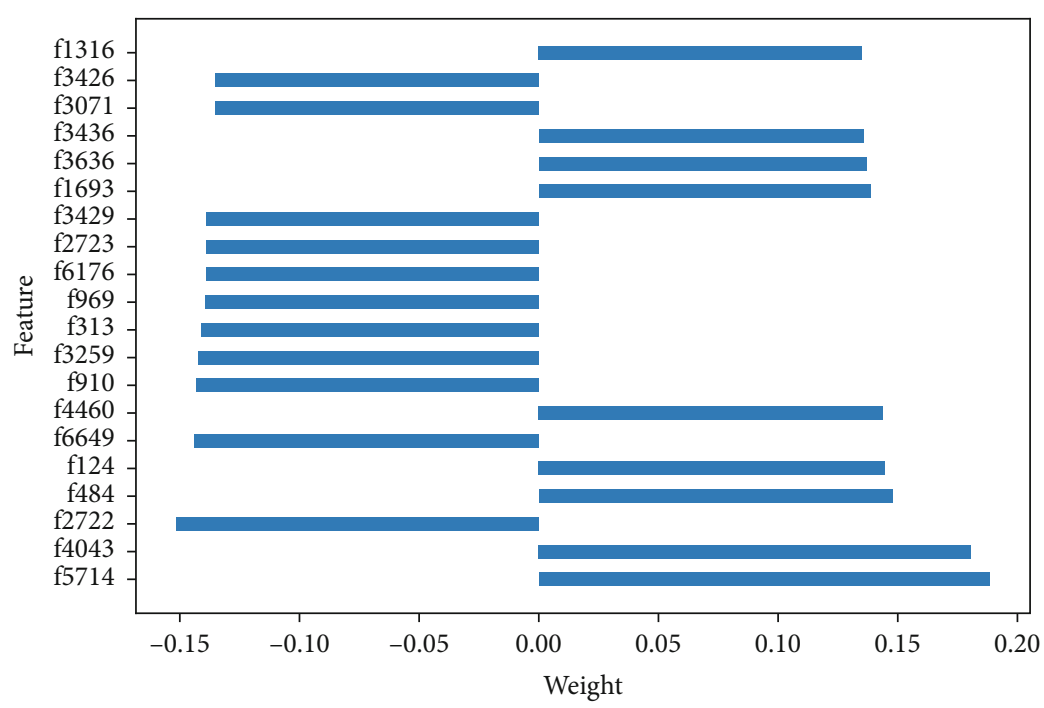

FIGURE 4: Weight visualization of some features of an instance.

formulated as a linear classifier with a fixed instance:

$$
g(x)=\widehat{W} x=w_{1} x_{1}+w_{2} x_{2}+\cdots+w_{n} x_{n} .
$$

Since the number of layers $L$ is 4 for the FCNN model we used in this paper, so the weight vector $\widehat{W}$ can be computed as

$$
\widehat{W}=\left(W^{(3)} \circ r^{(3)} \circ \tilde{\gamma}^{(3)}\right)\left(W^{(2)} \circ r^{(2)} \circ \tilde{\gamma}^{(2)}\right) W^{(1)} .
$$

The trained model could be interpreted with linear formula for any instance. Given an instance, we can get the weight of each feature from the trained model according to Equations (10) and (11). Some feature weights of an instance are visualized in Figure 4 . The vertical axis represents the feature index, and the horizontal axis represents the weight value. It can help to understand the prediction result according to the feature index which can correspond to the brain region involved in the feature.

5.2. Model Interpreting for the ASD Group. Based on the trained FCNN model, we used Algorithm 1 as described in Section 3.3 to extract the decision features of the model. We set the top-important feature parameter $K$ from 5 to 300 , with an interval of 5 , and the parameter $\varepsilon$ as $95 \%$, and then, we get a set of decision features with different $K$.

5.2.1. Decision Feature Evaluation. To evaluate the quality of the decision features, we analyzed the FCNN model by setting the values of the decision features in instance $x$ to zero and observed the changes of prediction of FCNN. We used metrics including sensitivity, accuracy, and the change of prediction probability (CPP) which is the absolute change of probability of classifying $x$ as a positive instance, the number of label-changed instance (NLCI) which is the number of instances whose predicted label changes after being hacked.
For comparison, we also used the top $N$ weighted features of linear-SVM to hack linear-SVM. The results are shown in Figure 5. It is shown that average CPP of FCNN is higher, and the NLCI of FCNN can be more than SVM with more decision features. And FCNN has considerable performance in sensitivity and accuracy.

For further comparison, we also applied the popular locally linear interpretation method (LIME) [27] to get the decision features in the trained FCNN model. Similar to Algorithm 1 in Section 3.3, we obtain the top $K$ important features of each instance, and then, we obtain the decision feature set $F$ by selecting the feature $f$ whose occurrence number as a percentage of total instances is greater to the parameter $\varepsilon$. We set the same parameters ( $K$ from 5 to 300 , with an interval of 5 , and the parameter $\varepsilon$ as $95 \%$ ), and we did not obtain any decision feature. What is more, when we loosed the parameter $\varepsilon$ to $20 \%$, we also did not get any one feature. It means that the top 300 important features of the instance obtained by the LIME method are very different between instances in this model.

5.2.2. Decision Feature Analysis. When $K$ is taken as 20,15 decision features were obtained; we selected these 15 decision features as a case for further analysis. There are 23 brain regions (ROIs) of the AAL atlas that involved these $15 \mathrm{rsFC}$ connections. These $15 \mathrm{rsFCs}$ and 23 ROIs are shown in Table 2.

We computed the mean value of each rsFC of the ASD group and the $\mathrm{HC}$ group, respectively, as well as the mean difference of two groups. An independent two-sample $t$ test was run on the means of the rsFC elements of two groups. The analysis is shown in Table 2. Among these 15 rsFCs, 2 rsFCs are statistically significant $(p<0.05)$ between the ASD and HC groups, and the rest of rsFCs are not statistically significant. It demonstrates that FCNN could find underlying features though the feature values are not statistically different between groups. 


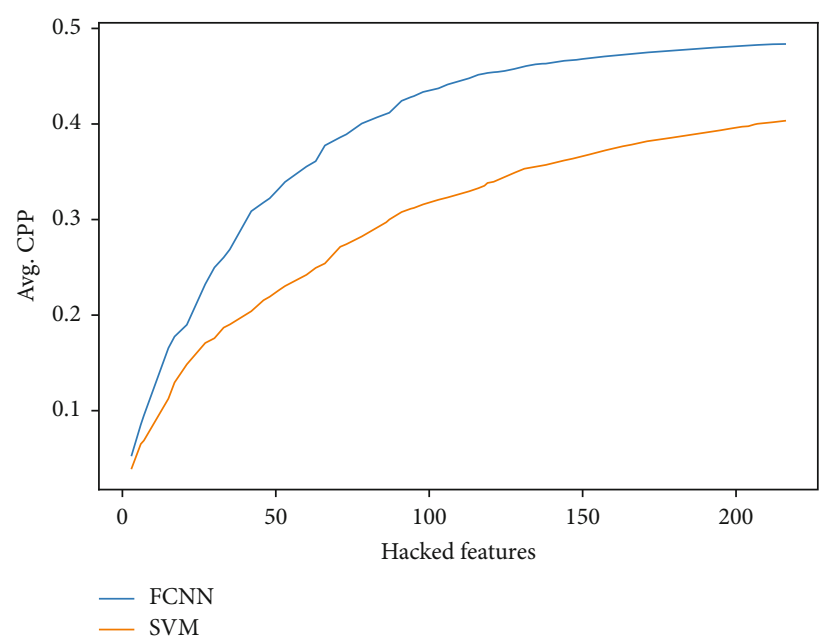

(a)

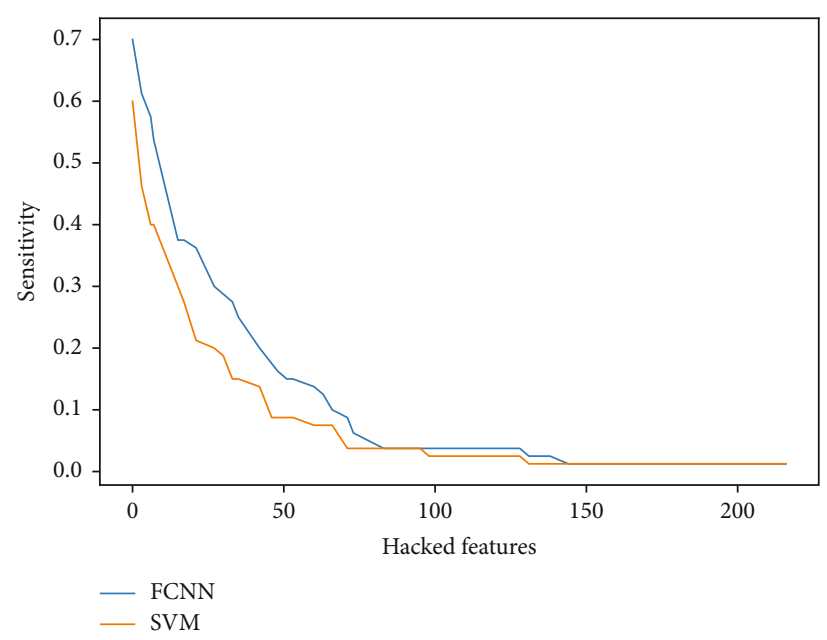

(c)

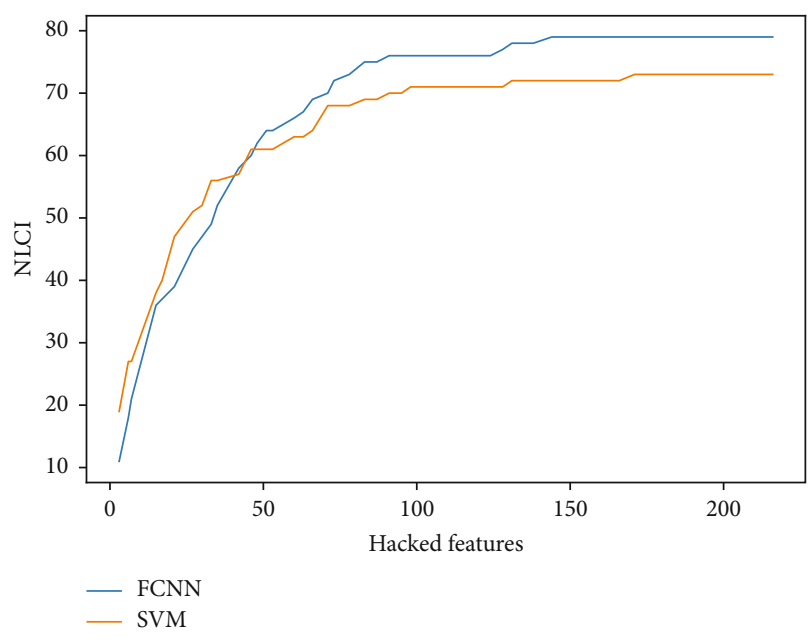

(b)

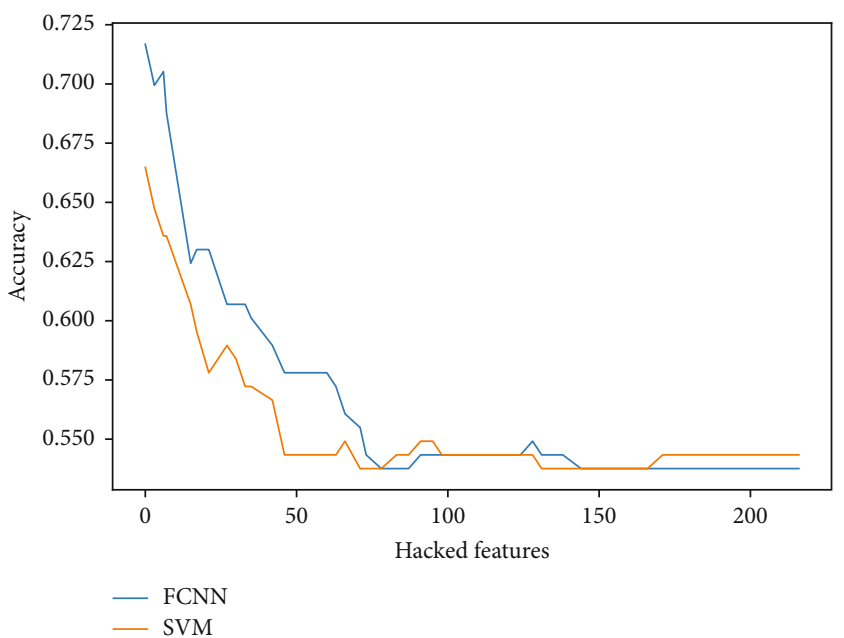

(d)

Figure 5: The performance of decision features on FCNN and SVM.

These 15 rsFC connections of the AAL atlas are visualized in Figure 6, where the label information is from the AAL atlas. The thicker connection indicates two regions are strongly correlated and vice versa. The figure was drawn with BrainNet Viewer [28] software.

5.2.3. Impact of Parameter $\varepsilon$. In order to evaluate the influence of parameter $\varepsilon$ on the obtained decision features, we set the parameter $K$ from 5 to 300 , with an interval of 5 , and the parameter $\varepsilon$ from $70 \%$ to $95 \%$, with an interval of $5 \%$; then, $N$ decision features were obtained accordingly. The result is shown in Figure 7. It is clear that the smaller the parameter $\varepsilon$, the more decision features will be obtained. While with a fixed $K$, the bigger the parameter $\varepsilon$, the fewer the decision features will be obtained.

\section{Conclusion and Discussion}

In this paper, we introduce an interpretable learning approach for resting-state functional connectivity analysis.
We firstly propose an interpretable FCNN to classify ASD from $\mathrm{HC}$, based on rsFC features. We experimentally compared the FCNN model against widely used classification models including SVM, RF, and two new classes of deep neural network models in a large dataset containing 871 subjects from ABIDE I database. The results show the proposed FCNN model achieves the highest classification accuracy $69.81 \%$.

We further propose an interpreting method which could explain the trained model with a precise linear formula for each input instance and identify decision features of the model which contributed most to the classification of ASD versus HC participants.

Though being focused on ASD analysis in this presentation, the proposed approach could be generalized to benefit many other brain science and medicine applications that involve deep neural networks. Particularly, this study offers a promising deep learning-based approach to explore potential biomarkers for assisting brain neurological disorder diagnosis and research. 
TABLE 2: Analysis of 15 most significant rsFCs.

\begin{tabular}{|c|c|c|c|c|c|c|}
\hline Connection ID & ROI number & Regions & ASD mean conn & Control mean conn & Mean difference & $p$ value \\
\hline \multirow{2}{*}{1} & 72 & Caudate_R & \multirow{2}{*}{0.0919} & \multirow{2}{*}{0.0728} & \multirow{2}{*}{0.0192} & \multirow{2}{*}{0.4390} \\
\hline & 107 & Cerebelum_10_L & & & & \\
\hline \multirow{2}{*}{2} & 44 & Calcarine_R & \multirow{2}{*}{0.7370} & \multirow{2}{*}{0.7256} & \multirow{2}{*}{0.0114} & \multirow{2}{*}{0.4920} \\
\hline & 46 & Cuneus_R & & & & \\
\hline \multirow{2}{*}{3} & 2 & Precentral_R & \multirow{2}{*}{0.5996} & \multirow{2}{*}{0.5474} & \multirow{2}{*}{0.0522} & \multirow{2}{*}{0.0325} \\
\hline & 12 & Frontal_Inf_Oper_R & & & & \\
\hline \multirow{2}{*}{4} & 50 & Occipital_Sup_R & \multirow{2}{*}{0.7175} & \multirow[b]{2}{*}{0.7136} & \multirow[b]{2}{*}{0.0038} & \multirow[b]{2}{*}{0.8445} \\
\hline & 52 & Occipital_Mid_R & & & & \\
\hline \multirow{2}{*}{5} & 5 & Frontal_Sup_Orb_L & \multirow[b]{2}{*}{0.2666} & \multirow{2}{*}{0.2309} & \multirow[b]{2}{*}{0.0357} & \multirow[b]{2}{*}{0.1985} \\
\hline & 36 & Cingulum_Post_R & & & & \\
\hline \multirow{2}{*}{6} & 16 & Frontal_Inf_Orb_R & \multirow{2}{*}{0.4435} & \multirow{2}{*}{0.4311} & \multirow{2}{*}{0.0124} & \multirow{2}{*}{0.6172} \\
\hline & 90 & Temporal_Inf_R & & & & \\
\hline 7 & 13 & Frontal_Inf_Tri_L & 04210 & 04102 & 00027 & \\
\hline & 16 & Frontal_Inf_Orb_R & 0.4219 & 0.4192 & 0.0027 & 0.9201 \\
\hline 8 & 6 & Frontal_Sup_Orb_R & 05250 & 04080 & הברח & ז-ברט \\
\hline 8 & 26 & Frontal_Med_Orb_R & 0.5359 & 0.4989 & 0.0371 & 0.2355 \\
\hline 9 & 44 & Calcarine_R & 06017 & 06632 & & 02427 \\
\hline 9 & 50 & Occipital_Sup_R & 0.6847 & 0.6632 & 0.0215 & 0.3427 \\
\hline 10 & 64 & SupraMarginal_R & 0.3853 & 0.3586 & 00267 & 0.3485 \\
\hline & 69 & Paracentral_Lobule_L & & & & \\
\hline 11 & 38 & Hippocampus_R & 02871 & 02618 & 00253 & 03651 \\
\hline 11 & 66 & Angular_R & $0.20 \% 1$ & 0.2010 & 0.0253 & 0.5031 \\
\hline 12 & 36 & Cingulum_Post_R & 02296 & 02110 & 00185 & 0.5694 \\
\hline & 43 & Calcarine_L & & & & \\
\hline 13 & 36 & Cingulum_Post_R & 02610 & 02474 & 0167 & 6090 \\
\hline 13 & 44 & Calcarine_R & & & & \\
\hline 14 & 38 & Hippocampus_R & 04710 & 04123 & 00586 & 00377 \\
\hline 14 & 86 & Temporal_Mid_R & & & 0.0380 & $0.03 / 11$ \\
\hline 15 & 68 & Precuneus_R & 0.3425 & 0.3111 & 0.0314 & 0.2958 \\
\hline & 81 & Temporal_Sup_L & & & & \\
\hline
\end{tabular}

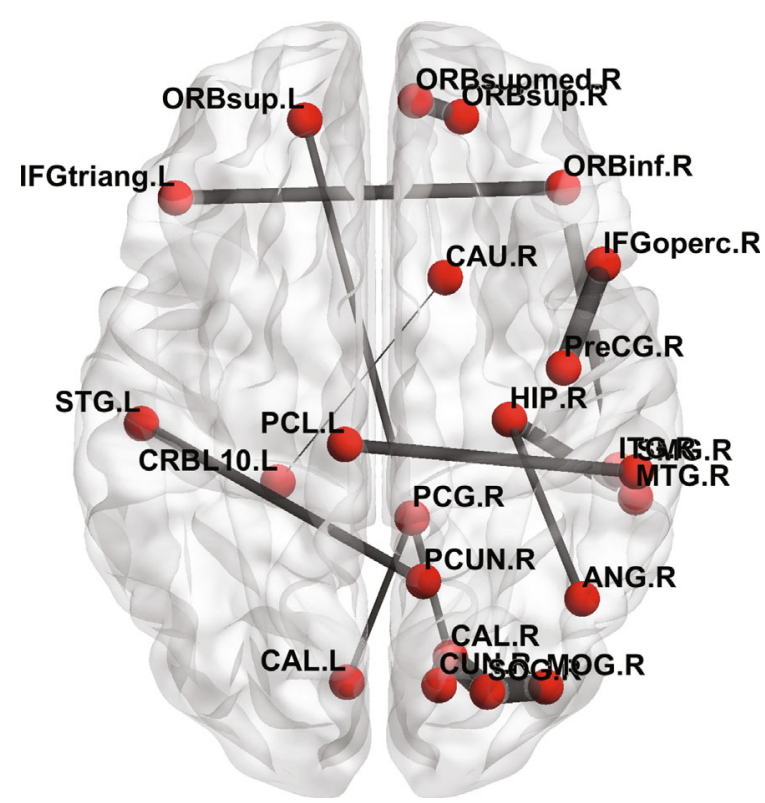

FIgURE 6: The visualization of 15 rsFCs from the ASD group. 


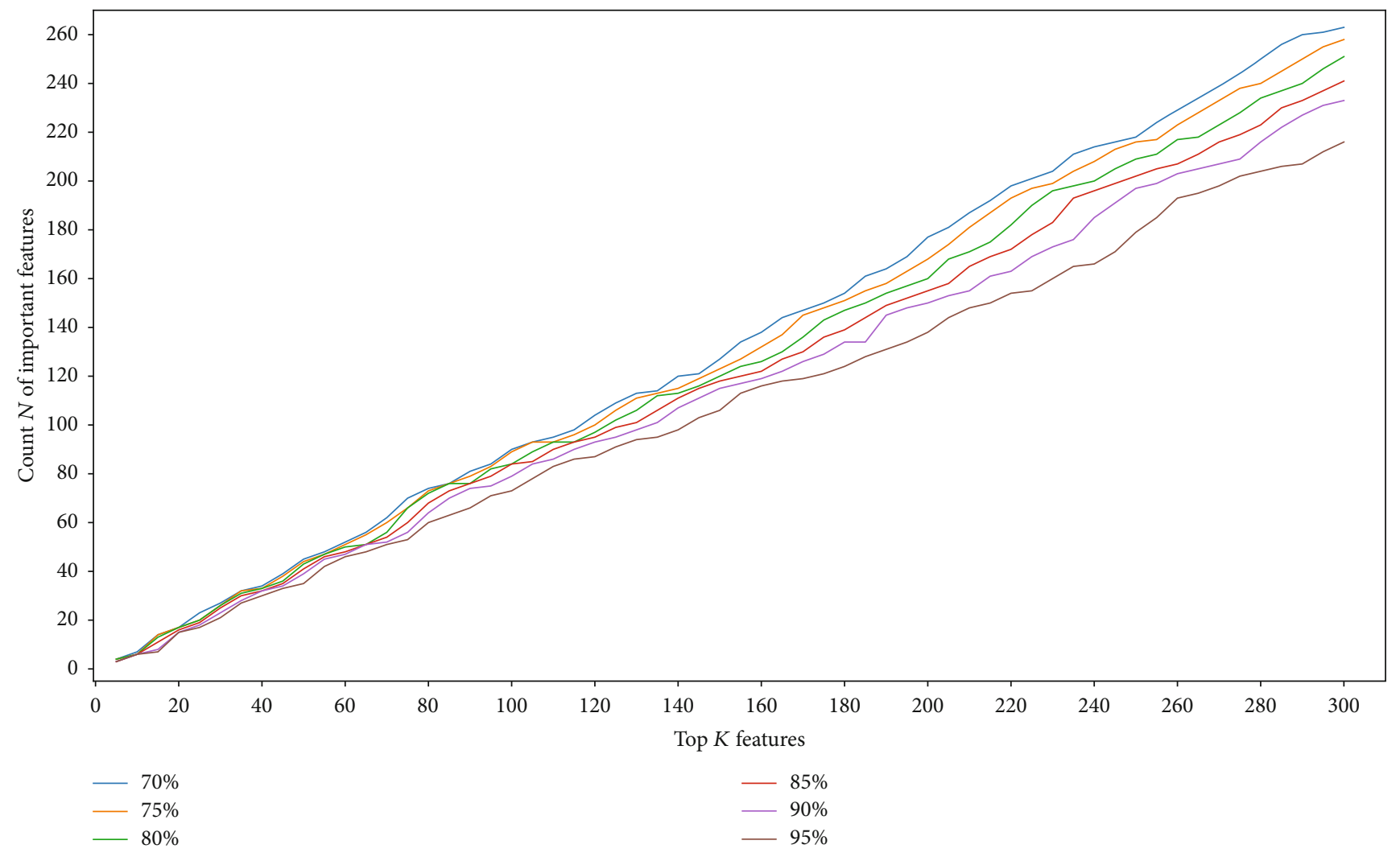

Figure 7: The number of decision features with different $K$ and $\varepsilon$.

There are two limitations in the current work presented here. First, the dataset is limited to the 871 participants that contained ASD and HC. In order for this work to be more generalizable, it would be important to inspect and compare these initial findings with more fMRI data from more participants. Second, the proposed model is a compact fully connected neural network, given the number of layers and nodes in the model. Thus, it would be important to inspect the effectiveness of our interpreting approach for other types of neural network such as deeper and more complex architectures in the deep learning literature. Future work should focus on the accuracy and interpretation of our proposed approach for other large-scale fMRI data as well as other neuroimaging data based on brain disorders such as ASD.

\section{Data Availability}

The data used to support the findings of this study are available from the corresponding author upon request. The ABIDE I dataset analyzed during this study is available in the Preprocessed Connectomes Project website (http:// preprocessed-connectomes-project.org/abide/download .html).

\section{Conflicts of Interest}

The authors have nothing to disclose.

\section{Acknowledgments}

This work was supported in part by the Natural Science Foundation of Guangdong Province of China (grants \#2018A030313309 and \#2015A030308017), the Innovation Fund of Introduced High-End Scientific Research Institutions of Zhongshan (grant \#2019AG031), the Fundamental Research Funds for the Central Universities, SCUT (grant \#2019KZ20), and the Guangdong Pearl River Talents Plan Innovative and Entrepreneurial Team (grant \#2016ZT06S220).

\section{References}

[1] C. M. Freitag and T. A. Jarczok, "Autism spectrum disorders," in Psychiatric Drugs in Children and Adolescents, pp. 383-403, Springer, Vienna, 2014.

[2] F. Volkmar, E. H. Cook, J. Pomeroy, G. Realmuto, and P. Tanguay, "Practice parameters for the assessment and treatment of children, adolescents, and adults with autism and other pervasive developmental disorders," Journal of the American Academy of Child \& Adolescent Psychiatry, vol. 38, no. 12, pp. 32S-54S, 1999.

[3] S. Takerkart, G. Auzias, B. Thirion, and L. Ralaivola, "Graphbased inter-subject pattern analysis of fMRI data," PLoS One, vol. 9, no. 8, p. e104586, 2014.

[4] R. Bhaumik, A. Pradhan, S. Das, and D. K. Bhaumik, "Predicting Autism Disorder Using Domain-Adaptive Cross-Site Evaluation," Neuroinformatics, vol. 16, no. 2, pp. 197-205, 2018. 
[5] H. Huang, D. Shen, and N. Carolina, "Enhancing the representation of functional connectivity networks by fusing multiview information for autism spectrum disorder diagnosis," Human Brain Mapping, vol. 40, no. 3, pp. 833-854, 2018.

[6] M. Plitt, K. A. Barnes, and A. Martin, "Functional connectivity classification of autism identifies highly predictive brain features but falls short of biomarker standards," NeuroImage: Clinical, vol. 7, pp. 359-366, 2015.

[7] M. A. Just, V. L. Cherkassky, A. Buchweitz, A. Timothy, and T. M. Mitchell, "Identifying autism from neural representations of social interactions : neurocognitive markers of autism," PLoS One, vol. 9, no. 12, pp. 1-22, 2014.

[8] B. Cao, X. Kong, and P. S. Yu, "A review of heterogeneous data mining for brain disorder identification," Brain Informatics, vol. 2, no. 4, pp. 253-264, 2015.

[9] A. Sólon, A. Rosa, R. C. Craddock, A. Buchweitz, and F. Meneguzzi, "Identification of autism spectrum disorder using deep learning and the ABIDE dataset," NeuroImage: Clinical, vol. 17, pp. 16-23, 2018.

[10] X. Guo, K. C. Dominick, A. A. Minai, H. Li, C. A. Erickson, and L. J. Lu, "Diagnosing autism spectrum disorder from brain resting-state functional connectivity patterns using a deep neural network with a novel feature selection method," Frontiers in neuroscience, vol. 11, 2017.

[11] T. Eslami, V. Mirjalili, A. Fong, A. Laird, and F. Saeed, "ASDDiagNet: a hybrid learning approach for detection of autism spectrum disorder using fMRI data," Frontiers in Neuroinformatics, vol. 13, pp. 1-8, 2019.

[12] C. J. Brown, J. Kawahara, and G. Hamarneh, "Connectome priors in deep neural networks to predict autism," in 2018 IEEE 15th International Symposium on Biomedical Imaging (ISBI 2018), pp. 110-113, Washington, DC, USA, April 2018.

[13] R. Anirudh and J. J. Thiagarajan, "Bootstrapping graph convolutional neural networks for autism spectrum disorder classification," in ICASSP 2019-2019 IEEE International Conference on Acoustics, Speech and Signal Processing (ICASSP), pp. 3197-3201, Brighton, United Kingdom, May 2019.

[14] X.-a. Bi, Y. Liu, Q. Jiang, Q. Shu, Q. Sun, and J. Dai, “The diagnosis of autism spectrum disorder based on the random neural network cluster," Frontiers in human neuroscience, vol. 12, 2018.

[15] A. Di Martino, C.-G. Yan, Q. Li et al., "The autism brain imaging data exchange: towards a large-scale evaluation of the intrinsic brain architecture in autism," Molecular Psychiatry, vol. 19, no. 6, pp. 659-667, 2014.

[16] J. Kawahara, C. J. Brown, S. P. Miller et al., "BrainNetCNN: convolutional neural networks for brain networks; towards predicting neurodevelopment," NeuroImage, vol. 146, pp. 10381049, 2017.

[17] X. Li, N. C. Dvornek, J. Zhuang, P. Ventola, and J. S. Duncan, "Brain biomarker interpretation in ASD using deep learning and fMRI," in International Conference on Medical Image Computing and Computer-Assisted Intervention, pp. 206-214, Springer, Cham, 2018.

[18] X. Li, N. C. Dvornek, Y. Zhou, J. Zhuang, P. Ventola, and J. S. Duncan, "Efficient interpretation of deep learning models using graph structure and cooperative game theory: application to asd biomarker discovery," in International Conference on Information Processing in Medical Imaging, pp. 718-730, Springer, Cham, 2019.
[19] L. Chu, X. Hu, J. Hu, L. Wang, and J. Pei, "Exact and consistent interpretation for piecewise linear neural networks: a closed form solution," Proceedings of the 24th ACM SIGKDD International Conference on Knowledge Discovery \& Data Mining, pp. 1244-1253, 2018.

[20] P. L. Bartlett, N. Harvey, C. Liaw, and A. Mehrabian, "Nearlytight VC-dimension and pseudodimension bounds for piecewise linear neural networks," Journal of Machine Learning Research, vol. 20, no. 63, pp. 1-17, 2019.

[21] N. Tzourio-Mazoyer, B. Landeau, D. Papathanassiou et al., "Automated Anatomical Labeling of Activations in SPM Using a Macroscopic Anatomical Parcellation of the MNI MRI Single-Subject Brain," NeuroImage, vol. 15, no. 1, pp. 273-289, 2002.

[22] A. Abraham, M. P. Milham, A. di Martino et al., "Deriving reproducible biomarkers from multi-site resting-state data: An Autism-based example," NeuroImage, vol. 147, pp. 736$745,2017$.

[23] C. Cameron, B. Yassine, C. Carlton et al., "The Bureau Preprocessing Initiative: open sharing of preprocessed neuroimaging data and derivatives," Frontiers in Neuroinformatics, vol. 7, no. 4, pp. 1-19, 2013.

[24] V. Nair and G. E. Hinton, "Rectified linear units improve restricted Boltzmann machines," ICML 2010 - Proceedings, 27th International Conference on Machine Learning, pp. 807$814,2010$.

[25] A. L. Maas and A. Y. Ng, "Rectifier nonlinearities improve neural network acoustic models," ICML Workshop on Deep Learning for Audio, Speech and Language Processing, 2013.

[26] S. Ioffe and C. Szegedy, "Batch normalization: accelerating deep network training by reducing internal covariate shift," International Conference on Machine Learning, pp. 448-456, 2015.

[27] M. T. Ribeiro, S. Singh, and C. Guestrin, “"Why should I trust you?" Explaining the predictions of any classifier," Proceedings of the 22nd ACM SIGKDD international conference on knowledge discovery and data mining, pp. 1135-1144, 2016.

[28] M. Xia, J. Wang, and Y. He, "BrainNet Viewer: a network visualization tool for human brain connectomics," PLoS One, vol. 8, no. 7, 2013 . 